Voix et Images

\title{
L'intimité allusive ou le simulacre biographique
}

\section{Jean-Pierre Vidal}

Volume 13, numéro 2 (38), hiver 1988

Le propre du corps Roger Des Roches

URI : https://id.erudit.org/iderudit/200708ar

DOI : https://doi.org/10.7202/200708ar

Aller au sommaire du numéro

Éditeur(s)

Université du Québec à Montréal

ISSN

0318-9201 (imprimé)

1705-933X (numérique)

Découvrir la revue

Citer cet article

Vidal, J.-P. (1988). L'intimité allusive ou le simulacre biographique. Voix et Images, 13(2), 258-269. https://doi.org/10.7202/200708ar d'utilisation que vous pouvez consulter en ligne.

https://apropos.erudit.org/fr/usagers/politique-dutilisation/ 


\title{
L'intimité allusive ou le simulacre biographique
}

\author{
par Jean-Pierre Vidal, Université du Québec à Chicoutimi
}

a Céline D.

Le maitre dont l'oracle est celui de Delphes ne dit ni ne cache mais donne des signes.

Héraclite, Fragments, 39 (traduction de Marcel Conche).

Un suspens retarde encore le siècle proche. La littérature, à n'en pas douter, s'y dérobe dans le fracas médiatico-industriel des grands catafalques du futile: téléromans, best-sellers, romans dits populaires revendiqués par ceux des universitaires qui prétendent encore, par cette démagogique élection, à l'on ne sait quelle miraculeuse indépendance de l'institution qui pourtant là encore, là surtout, les point, toute une écriture grasse et veulement narrative encombre nos lectures. Dans ce qui reste d'autre, au désert, l'aphorisme se cisèle précieusement, comme pour mieux refuser tout commerce avec la complaisance aveugle des lectures de cette fin de siècle languissante.

C'est le temps dès lors des célibataires, écrivains au secret. C'est le temps, mais nul presque ne le sait, des écrivains résistants. C'est le temps de Des Roches.

Et cela dure depuis vingt ans demain.

Non qu'il soit ni le seul, ni le plus ancien, de nos ermites émérites. Mais il est, fidèlement, irrésistiblement, le plus seul. Parce que toute son œuvre tient au texte des autres. Et c'est en quoi, du reste, il demeure, modestement, le plus moderne encore. Par la hantise de l'ouverture, de l'impossible trouée de la parole. Car jamais parole n'a franchi le seuil des corps disait déjà l'épigraphe, empruntée à Tzara, du tout premier recueil: Corps accessoires 1 . Des Roches, l'explorateur des seuils, le parcoureur de lisières, et plus encore: l'ouvreur de sas. D'une parole à l'autre, d'un corps à ce qui se médit pour sa parole, de ce qui se dédie d'un

1 Les Éditions du Jour, Montréal, 1970, 55 p., repris dans «Tous, corps accessoires...» (poèmes et proses, 1969-1973), Montréal, les Herbes rouges, $1979,292 \mathrm{p}$. C'est cette dernière édition que nous utiliserons pour toutes les références faites, dans cet article, au recueil Corps accessoires. 
texte à ce qui s'emporte, toujours vain, dans la fureur vite d'une écriture à l'affût.

Je parlerai donc, ici, en guise d'ouverture, d'une forme de béance, d'une manière d'appétit et d'une urgence qui n'a de cesse.

\section{Portrait de l'artiste en jeune Don Juan}

Qu'Apollinaire et Joyce nous pardonnent ce liminaire jumelage de leurs titres. Il dira, à leur enseigne, les commencements d'un avide de littérature, de la littérature considérée comme un des beaux artifices de la reconnaissance (voir ici même l'entretien de Roger Des Roches avec André Gervais). Car, en effet, d'emblée l'acte d'écrire est pour Des Roches un appel et une effraction, une adresse pourrait-on mieux dire. Adresse à l'Autre, celui, traditionnellement majuscule de la philosophie, dont le texte ou l'écriture ne sont que des figures.

\section{Et d'emblée le texte de Des Roches en est tout envahi.}

Éperdument toumée vers cet improbable dehors, l'écriture est du même coup ployée vers une intimité qu'elle pose comme un don, comme l'objet même du don: celui qu'elle se fait être vers l'autre. Écrire, c'est toujours, quelque peu, feindre l'intimité en posant, a priori, l'autre comme son écoute.

Écrire, donc, c'est occuper l'espace de la liaison en sa visée, ses fins. En texte, il ne sera dès lors question que d'étreintes toujours défaites, de ruptures et de deuil célibataire. Corps tombés, disjoints, les «corps accessoires» dont se titre le premier recueil sont des corpuscules que l'écriture résolument synecdochique de Des Roches éparpille en des fictions d'images cubistes où bien malin qui pourrait faire prendre corps. Dès le début de l'œuvre, en effet, ça se divise, grouille et proliferre.

Mais le désir est encore bien littéraire. Il est, je l'ai dit, désir de la littérature comme lieu de la liaison proposée et acceptée, il faudrait dire, même, «bien reçue», au sens qui dénote le message en ondes, car c'est bien d'ondes ou de souffle qu'il s'agit. Écrire, en ces débuts rimbaldiens, c'est mimer la geste thématique obligée de l'adolescence (je me retrouve sans ces chairs / dans des psaumes étranges chantant le mal comme on chante une patrie. Corps accessoires, p. 15), pour que quelque Verlaine, mais presque aussi jeune que soi et qui pourrait s'appeler Denis Vanier, réponde, du haut de la littérature: ...Venez, chère grande âme, on vous appelle, on vous attend2. Au fond, Des Roches écrit alors à la littérature...

Et les corps de femmes, multiples, explorés en détails jamais rassemblés, qui hantent la fiction de tous poèmes, ce ne sont que fantasmes divers où se dit le rêve littérature, c'est-à-dire rien, rien d'autre que la pulsion d'écrire.

2 Lettre de Verlaine à Rimbaud (septembre 1871), Euvres complètes de Rimbaud, Paris, Gallimard, «Bibliothèque de la Pléiade», 1963, p. 281. 
Et comme l'adolescent n'a jamais le temps d'apprendre le corps de l'autre, pas plus que le sien propre, mais plus précipité encore ici le mime du désir parce que le corps de la littérature, son corpus, est un insaisissable, Des Roches n'a guère le temps de vérifier, comme on dit, ses sources, ni même qu'il y a quelque part hors de lui ce lieu improbable qu'on pourrait dire source. Des Roches ne sait pas citer, parce qu'il ne sait pas, ne veut pas, ordonner. En fait, carrément, il charrie, il charrie des bribes de textes qui lui (nous) viennent de partout. Et s'il ne sait pas ordonner, il sait du moins qu'il cite, toujours, comme quiconque; il sait, et admirablement, comme personne, moduler. Il ne rendra jamais aux césars surréalistes (entre autres) ce qui de lui leur revient, car la monnaie qu'ils ont battue, il la fait, lui, Des Roches, circuler, il en prolonge le cours. Texte toujours de l'urgence, fait de fragments arrachés on ne saura plus d'où, l'œuvre tout entière de Des Roches est un intertexte vite. Comme déjà elle l'était pour-Lautréamont (dont bien souvent le texte croise le sien), la citation est son multiplicande.

C'est pourquoi d'ailleurs cette œuvre tient de tous ses rythmes au paysage urbain. C'est pourquoi elle est joycienne en fin de compte, même si nul roman encore ne s'y déploie. L'aspect nord-américain du perpétuel vite, du multiple intermittént de la cité (tous thèmes aussi de la «Nouvelle Écriture» mais qui sont encore déjà surréalistes. Trouve-t-on une histoire avant de trouver un lieu?), tout ce charivari de la rue et des bars est un peu, comme la femme imaginaire (ou le corps de l'autre qui, quelque part dans le surgissement du tracé, n'a jamais de sexe discernable), la figure du texte. Car chez Des Roches la fiction est métarhétorique plus que, banalement selon l'époque, métalangagière 3 . Voilà pourquoi, d'ailleurs, il est, un des seuls, encore moderne aux temps actuels du simulacre.

«Les aimer toutes», ce précepte donjuanesque qui court, explicite (dans l'Imagination laïque et Tout est normal, tout est terminé, notamment) ou à peine tu, d'un texte à l'autre, vise plus des graphes, des paroles, des écritures, des intensités de greffes que le (corps) féminin tout entier, ou même le corps (parfois féminin), à supposer que la féminité se puisse saisir autrement que, selon le mot de Hegel, comme l'éternelle ironie de la communauté, c'est-àdire comme négativité, détour, rupture, décrochage... du sein même de qui (la communauté du phallus? Le désir lui-même?) la profêre. Et, bien sûr, cela n'exclut pas qu'il y ait aussi toujours du biographique, notre seul «réel», et que ce biographique cherche toujours à s'inscrire selon maintes insaisissables feintes. Mais, comme l'énonce si bien Normand de Bellefeuille, ce vieux complice et sans doute jumeau d'écriture de Des Roches, il y a:

\section{Cette ćertitude presque, malgré toutes les théories, qu'il n'y a rien qui}

3 C'est-à-dire que cette fiction ne devient que rarement, par mise en abyme ou quelque autre tour, illustrative, fût-ce de ses modes de production. En ce sens, elle ne fait rien savoir, elle ne fait pas signe de savoir. Elle est seulement la rhétorique du texte se prenant pour cible ou crible, se perpétuant dans une relève (Aufhebung) qui est autre. Elle est, si l'on veut, la négativité (au sens hégélien encore) de l'interminable processus dit texte. C'est ce processus que j'appelle la rhétorique du texte. 
préexiste d̀ l'écriture, que la pulsion ne préexiste pas à la phrase, que c'est celle-ci, bien au contraire, qui l'engendre à l'instant même de l'inscription.

\section{L'écriture est avant tout initiative 4 .}

La voix qui se signe Des Roches savait sans doute obscurément, en ses premiers souffles, qu'il en est bien ainsi et que rêver par exemple la Femme ou dire par exemple l'amante biographique, c'est d'abord rêver et dire, rêver de dire, son écriture, la sienne propre et celle de l'Autre. Seul véritable tabou du texte, c'est là précisément ce qui ne peut șe dire et par quoi ça s'écrit. Car si je pouvais dire mon écriture, (c'est qu') elle n'aurait pas lieu.

D'où cette impression, forte, étrange et vraie:

Si je découvrais aujourd'hui

Que j'écris le même livre depuis bientôt vingt ans? 5

Et encore ceci:

Car j'écris à une femme.

$J^{\prime} e ́ c r i s$ toujours à une femme.

J'écris toujours à la même femme ceci:

"Je t'ai choisie au hasard,

Parmi toutes les femmes qui vont disparaître.

Avant que tu ne deviennes mon ennemie.»6

Qui ne voit qu'ici «j'écris» et «femme» s'inscrivent dans cet espace de la quasi-synonymie que seul le tracé, l'inscription déploie, paradigme syntagmatisé (selon une autre formulation teńtée ici du «principe poétique» de Jakobson), ouverture au cœur du même, différance derridienne 7 à n'en pas douter.

Dès ses bruissements premiers, ses initiales inscriptions, le texte Des

4 La Mort du genre, actes du colloque tenu à Montréal en octobre 1987, la Nouvelle Barre du jour, $n^{\circ}$ s 209-211, 1987, p. 86.

5 Roger Des Roches, Tout est normal, tout est terminé, Montréal, les Herbes rouges, $n^{\circ} 160,1987$, p. 22.

6 Ibid., p. 10.

7 À noter qu'avec une lucidité que même l'effet mode — indéniable alors, s'agissant d'un tel nom - ne saurait réduire, François Charron convoquait déjà Derrida dans sa préface à l'Enfance d'yeux, dès 1971.

De Bellefeuille, Charron, Louis-Philippe Hébert, André Roy, ces trois derniers conjointement dédicataires de Tout est normal..., Des Roches c'est aussi, décidément, une constellation à l'exception du lieu littérature. Il faudra un jour se préoccuper de l'intertexte que signent, au milieu des Herbes rouges et, plus généralement, de la Nouvelle Écriture, ces quelques noms qui ne sont certes pas des moindres. 
Roches est aux prises avec le bris, le barré, la coupure. Architextoniques, des strates mobiles de langue s'y rencontrent violemment, produisant ça et là, à la faille, des tremblements de texte, un feuilletage. Ou, comme l'écrit si justement François Charron: Les mots semblent s'inter-pénétrer, se crocheter dans la liaison 8 .

Le texte, indéfiniment, suture des bribes adverses, irrésistiblement (d'on ne sait où) advenues. C'est un autre de ses modes de dirè la «différance» qui le meut. Car son intimité se tient aux marges, là où le regard de l'autre (autant dire la lecture) la déploie dans la publicité de son dehors: la langue et plus encore la littérature comme citations déjouées.

Il faut donc maintenant l'y voir là où il y a casse.

\section{Un vague murmure segmenté9}

On nous permettra, puisque cet article prend l'œuvre entière par ses deux extrémités: son surgissement et sa dernière manifestation (Tout est normal, tout est terminé), et que celle-ci comme celui-là sont de l'espèce communément appelée «poésie», d'ignorer, et c'est aussi faute de temps, l'autre pôle: la prose.

De cette dernière d'ailleurs il manque pour le moment à la lecture le plus narratif sans doute de Des Roches: les pièces radiophoniques qu'il serait temps qu'un éditeur rassemble. De plus, c'est dans cet espace-là que se croise, signée, ressourcée, proposée à rebondir visiblement, l'œuvre identifiée de l'autre autorisé: Pourvu que ça ait mon nom 10 avec Normand de Bellefeuille. Ici encore, l'allusion à d'autres espaces littéraires se fait, sous les espèces de la $\mathrm{S}$. F., plus nette, tandis que le zébrage ligné des mots dans la page s'articule à d'étonnants dessins: Reliefs de l'arsenal11. Sur ce même espace prose, enfin, se projette ironiquement le fantasme d'un bon gros roman d'épouvante ou érotique (ce qui, dans ce contexte, cet intertexte, est la même chose), le best-seller défi, l'épais narratif relâché à opposer à l'intense scriptural lacunaire jusques à maintenant travaille.

Autant de raisons de surseoir à une prise en compte qui se devrait d'être

8 François Charron, préface à l'Enfance d'yeux suivi de Interstice, Montréal, Éd. du Jour, 1972, p. 10.

9 ... et un vague murmure segmenté rappelle aux poissons l'existence du téléphone dans: «l'Architexte dur», suite de poèmes repris dans Autour de Françoise Sagan indélébile: voir «Tous, corps accessoires...», op. cit.2p. 216.

10 Roger Des Roches et Normand de Bellefeuille, Pourvu que ça ait mon nom, Montréal, Éd. les Herbes rouges, 1979, (coll.«Lecture en vélocipède»), $71 \mathrm{p}$.

11 Roger Des Roches, Reliefs de l'arsenal, Montréal, l'Aurore, 1974, (coll. «Ecrire»), $93 \mathrm{p}$. 
globale pour faire sa juste place au divers.

Non que la «poésie» RDR ne soit elle-même fort diverse mais du moins son hybridation, son métissage se marquent-ils à l'intérieur d'un genre plus strictement balisé, d'un genre où l'existence ancestrale de formes fixes s'est, depuis maintenant au moins plus d'un siècle, combinée à l'exigence d'écarts plus absolus.

La poésie, donc, provisoirement.

Dans une «Note sur le travail formel» insérée dans la postface qu'il proposait à Autour de Françoise Sagan indélébile, François Charron, encore, écrivait, à propos d'un livre conjugant, il est vrai, poèmes et proses, ceci:

Le travail sur les signifiants est ici primordial. Je laisserai à l'auteur le soin de nous expliquer les subtilités de ses techniques, pour dire brièvement qu'il se dégage deux plans englobants: celui de la brisure et celui de l'allitération. Par brisure nous entendons arrêt subit et immotivé d'un mot ou d'une phrase 12 .

Bien dit, effectivement, ici encore, le mouvement de la poésie RDR obéit à deux phases, s'inscrit sur deux plans: le lacunaire (la «brisure» de Charron, qui est aussi une manière de pointillé à remplir) et le redoublé (l'allitération) ou, si l'on préfere, le manque et le même.

«Différance» encore, on l'aura du même souffle dit, différance à l'intérieur même du signifiant dont l'atome littéral à la fois pris et dégagé, lettre volante, insémine et fracture tout référent.

Car la lettre elle-même en effet sait s'y faire lire ou taire, entre deux abîmes: l'hébétude bègue du texte redoublé, pure surface, et l'hallucination quasi holographique du texte troué, perte ou volume.

Ainsi, dans l'Imagination lä̈que ${ }^{13}$, suite de 1475 vers numérotés et distribués en six «souffles», sans ponctuation, tel accident littéral, ses indices et sa répercussion: ici traite-t-on le muet comme il se doit (1er souffle, vers 390, p. 26) et, un peu plus bas, en reprise: ici traite-t-on le muet drague du muet (vers 398). D'emblee le redoublement des $t$, des traits même dirait-on, le troisième, prothèse qui évite mais fait donc percevoir le hiatus (Robert: 2 . vieilli. Solution de continuité, espace entre deux choses, dans une chose), les deux derniers en fin de mots et muets eux-mêmes alors que les deux premiers sont l'un

12 Autour de Françoise Sagan indélébile, Montréal, l'Aurore, 1975, (coll. «Lecture en vélocipède»), p. 97. Cette postface n'a malheureusement pas été reprise dans l'édition de l'œuvre parue dans *Tous, corps accessoires...».

13 Roger Des Roches, l'Imagination laïque, Montréal, Éd. les Herbes rouges, 1982, (Coll. «Lecture en vélocipède»), 78 p. 
à l'initiale, l'autre en milieu de mot, tous deux au contraire prononcés, ce redoublement des $t$ donc, et sa stratégie font que striée, clivée, la phrase est prête à se rompre, non seulement de part et d'autre du hiatus enjambé («trait», «te», «ton», voire même «téton» dans un texte où, comme toujours chez Des Roches, le sein est honoré) mais aussi et surtout dans «le muet» où s'entend, dès lors, par la possibilité d'une élision secrète: «le e muet».

Cette lettre cachée dans la lettre, puis draguée par une lecture dont on devrait suspendre le jugement d'arbitraire avant d'avoir lu plus bas, la voici bientôt ça et là ressurgie à la rime (toute de «e» muets, quand elle existe... C'est-à-dire, en fin de compte, toute d'absence sonore), la voici surtout dans telle suite ${ }^{14} \mathrm{du}$ «sixième souffle»:

quel ce dernier poème $e$

quel ce livre e grogne e est né d'une trop longue absence et l'absence $e$ continue à sa manière selon

(vers 1395-1397)

Et encore dans ce vers: comme la pensée muette e ou l'engagement muet e (vers 1415), où, sur et hors l'adjectif, elle fait effet de genre.

Or précisément, l'Imagination laïque tire maints effets du déni réglé du genre, au moins grammatical, mais grammatical pas exclusivement, puisque la bisexualité ou la parfaite ambiguïté sexuelle sont, comme on l'a maintes fois souligné (et notamment Charron 15 , encore), au mitan des fictions produites par le texte RDR et que, de la lettre au biographique ou au mythologique, le parcours s'effectue par l'intermédiaire du social et de la surcharge que le féminin produit maintenant dans tout texte qui prétend à un destinataire ou un allocutaire collectif.

Déni réglé du genre ces l'écriture est une silence (vers 318), un texte étanche aux larmes comme le raison (vers 353), mais rien n'existe de chaque côté de le travail monsieur (vers 383), dans le premier souffle, et autres là où le loi vit

14 Elle est déjà, en fait, apparue bien avant, flottante:

ainsi les mots bon sous la jupe ici nul ne souffre $e$

nul ne manque $e$ (vers 297-298),

juste après le fort étonnant :

pourquoi parce que l'amoureux mouille (vers 295).

Si bien qu'il aurait fallu, en toute rigueur (mais l'ordre de présentation du texte est-il l'ordre de son écriture et la lecture y est-elle contrainte?), attribuer aussi à la mobilité d'abord visible de cette lettre une part importante dans le processus de profération enfin de «l'e muet». De fait, c'est à la fois le «t» redoublé et le «e» envolé, toujours de trop, qui font venir cette nomination à la surface du signifiant: «le muet».

On invoquera, comme raison de ce passé sous silence de ces occurrences plus liminaires, les nécessités de l'analyse en son exposition.

15 Dans sa postface à Autour de Françoise Sagan indélébile, op. cit. 
indistinctement (vers 1429$)^{16}$, et de mon masculin rentrée dans la matière (vers 1439) ${ }^{17}$, dans le sixième.

En fait, c'est tout au long du texte que de telles dysgraphies se marquent, toutes prêtes d'ailleurs à rendre sens quand même dans le volume du lapsus: l'écriture est une science, étanche aux larmes comme de raison, etc.

Tout se passe donc comme si d'invisibles parenthèses devaient pouvoir de chaque vers autonomiser telle ou telle partie. Ainsi, par exemple, qu'en ces deux vers, du deuxième souffle: animer sans les mes ailes du sacré ou du profane (vers 665 ) et ceci comme les mes salaces figures (vers 676).

Lettres ou mots de trop, lapsus, parenthèses absentes, mots-valises (réconciliabule, vers 471; célébrataire, vers 1268, pour prendre des exemples dans les deux extrémités du texte), véritables trous noirs syntaxiques, ce texte est une cérémonie du fluide, le labile est son mode privilégié, le précipité son legs.

Volume et surface sans bornes, il fait souvent signe, par son agrammatisme, son absence de toute ponctuation du moins graphique, aux textes des débuts de Des Roches que, par ailleurs, véritable chanson de geste du typographe, il prend à revers, pseudo-haikus qu'ils sont parfois, de toute l'envergure de son épique allonge.

On n'écrit pas des poèmes, on les corrige dira, en italique, Tout est normal, tout est terminé (p. 24). Et c'est façon de dire qu'il n'y a jamais de fond, originel ou ultime, jamais de fin à l'écriture. Et qu'elle n'a jamais fini de convoquer son signataire, à travers les feintes intimités du «jeune écrivain» et de «la passante» (l'Imagination laique), ou de leur produit: «le célibataire», figure mythique par quoi, dans son dernier texte surtout, Des Roches, sans trop vouloir, semble-t-il, le savoir, salue ces deux grands piégeurs de la signifiance moderne: Lautréamont et Duchamp.

\section{Le célibataire mis à nu, mais}

Signe de Duchamp, et de sa «Mariée mise à nu par ses célibataires, même» d'abord dans le seul véritable agrammatisme qui ponctue Tout est normal, tout est terminé: la place étrange d'une virgule non avant mais après un «mais» qu'ainsi elle suspend.

Relevons-en les occurrences:

16 On n'aura garde d'oublier que dans ce vers, en outre, comme dans certains autres, le déni du genre se redouble d'un travail sur l'accent dont l'effacement ferait apparaitre l'altemative et l'article «correct»: «la ou le loi».

17 de ma masculine rentrée ou de mon masculin rentré: le texte suspend le choix dans la faute, scansion, rythme laissé à la lecture. 
Tout s'apprend mais, tout se gaspille mais, je me console. (p.7)

Le visage change mais, le visage change. (p.8)

La pensée change mais, la permission vient trop tard mais,

La transparence se prouve toujours plus facilement. (p. 11)

Une femme qui se déshabille très mal mais, bel et bien,

Dont la peau goûte toujours le savon. (p. 13)

La voix change mais, l'éternel amoureux déclare mais,

La Solitude change les draps plusieurs fois par mois. (p. 21)

Comme le locataire mais, comme celui qui insiste mais,

Comme la langue de famille,

Comme séduit par qu'est-ce qui pleure mais,

Comme mes discours sur la possibilité de régner pour aimer. (p. 25-26)

Comme un regard, même inattentif, sur ces extraits, permet de le constater, sauf en une occurrence (celle de la page 8), cette virgule, qui sépare le «mais» de la phrase adversative que normalement il introduit, prend effet d'une alternative. Puisque cette phrase adversative, ou celle qui en tient lieu, survient toujours après le deuxième «mais» et que, justement, il n'y a qu'une fois de troisième «mais», et qu'une fois seulement il n'y en a qu'un.

On voit bien le système: comme chez Lautréamont la parenthèse, le point d'orgue qu'est la virgule après «mais» identifie, à l'intérieur de l'ensemble du texte, un réseau particulier dont le regroupement des éléments qui le composent offre à la lecture de surprenantes constatations.

Comme si, en ce rythme particulier d'une ponctuation autre à l'intérieur d'un texte où la ponctuation reste très sage, très "conforme», se disait, précipité, matrice, quelque épure de l'ensemble.

Et d'abord le battement: Tout s'apprend / Tout se gaspille. La barre est ici, en place de l'alternative, avec qui elle fait «différance», le signe du contradictoire: [amasser] $\neq$ [dépenser], [prendre] $\neq$ [perdre], etc. Systole, diastole, au cœur, au souffle du jeune don juan comme du vieux célibataire, au niveau thématique, biographique ou mythique. Car il y est aussi, bien sûr, question de texte, du texte comme différance perpétuelle. Souvenons-nous: On n'écrit pas des poèmes, on les corrige...

Battement donc, qui répercute le tout est normal, tout est terminé du titre (c'est aussi, extraction habituelle à Des Roches, le deuxième vers du texte) en sa double connotation ancestrale du cri du guet (Tout est calme, bonnes gens, dormez en paix) et du soupir ultime du sacrifice (Tout est consommê). Tout s'inscrit, tout s'efface, tout continue, tout se termine, mais. 
Mais reste le gaspillage, le mouvement, le change, la vie ajouterait ironiquement le vieux célibataire. Dans le sous-texte ou l'intratexte formé des citations précédentes, changent successivement: le visage, la pensée, la voix, la Solitude. Tous lieux du souffle célibataire, lieux de l'Un du vieux Parménide, lieux même du $Y$ a d'l'Un du bon docteur Lacan18.

Reste aussi le «comme», espace ouvert du métaphorique, lieu de la langue, lieu du lieu (de l'Autre, cf. «le locataire», «la langue de la famille»).

Et puis, toujours, cette entêtante mariée mise à nu, même. Déjà, dans Corps accessoires, on pouvait lire: j'aime la magical bride (...) j'aime la beauteous bride / $j$ 'aime la theorical bride / j'aime la cautious bride (...) $j^{\prime}$ aurais aimé la virgin bride...19

Cette femme qui, dans notre «intratexte» ici, se déshabille très mal mais, elle est aussi, ailleurs dans Tout est normal, tout est terminé:

Cette jeune femme-là assise debout couchée,

Appuyée contre les rayons de la bibliothèque.

Mariée qui a les fesses roses et le ventre Roger. (p. 10)

Tableau formé d'une pose contre la culture, portant une femme nue, dont on signe le ventre du même signe adjectif commun («rose» - Rrose? - rouge «Roger») de la chute d'un nom propre, recto / verso. Et que fait-elle? Elle attend que je me gaspille ( 3 vers plus bas). Tout s'apprend mais, tout se gaspille mais, je me console.

Or le Célibataire apparait, pour la première fois nommé, dans la deuxième strophe 20 , dont il faut décidément citer tout le début:

J'ai écrit tous les titres,

Mais pas les bons livres au bon moment.

18 «De la jouissance» dans le Séminaire. Livre XX, Encore, Paris, Seuil, 1975, (coll. «le Champ freudien»), p. 12.

Je ne résiste pas, tant elle tient à l'ensemble du propos de cet article, au plaisir de citer, de la page 15 du même texte, cette phrase:

Et c'est bien là l'étrange, le fascinant, c'est le cas de le dire - cette exigence de l'Un, comme déjà étrangement le Parménide pouvait nous le faire prévoir, c'est de l'Autre qu'elle sort. Là où est l'être, c'est l'exigence de l'infinitude.

Suit une analyse de Don Juan! Ne voyez-vous pas que l'essentiel dans le mythe féminin de Don Juan, c'est qu'il les a une par une. (Ibid.)

19 «Tous, corps accessoires...», p. 32.

20 Tout est normal, tout est terminé est formé de 22 séquences de 10 à 30 vers chacune. Ces séquences, je crois juste de les nommer «laisses». Car, comme l'Imagination laĩque mais à la différence des poèmes du début (encore que ceux-ci, dans leur individualité, ne laissent pas de former collectivement un texte suivi, dans chaque recueil), Tout est normal, tout est terminé forme un seul poème. 
On écrit tellement mieux que moi.

D'ailleurs ils tiennent l'histoire, d'ailleurs je la visite, d'ailleurs j'y songe.

Je m'enlève de son chemin. Je me console.

"De retour.le Jour de la Science. *

Aujourd'hui, l'oreille collée sur le cadavre d'un voyeur,

Je sens le fond de miroir, les métaphores,

La poésie allumée, la poésie éteinte.

Je vois ma relation avec cette jeune femme comme une mission à

l'étranger.

Couché à côté de mes membres,

Je vieillis, je plante mes doigts,

Je me bourre le corps d'ouate (la Rupture en fera autant).

Célibataire afin de durer mon temps parmi les autres célibataires.

Le «je», le Célibataire ne s'y disent que dans la division, l'exclusion, la Rupture, formes aussi de quelque scissiparité secrète de l'Un: du titre au livre, de tous aux bons, d'une pratique à sa synchronie («au bon moment»), du «On» (qui n'exclut pas nécessairement mais uniquement parfois la personne qui parle) au moi, de l'histoire tenue à l'histoire visitée, de la publicité à l'intimité. L'intimité ici commence au pronominal21:je m'enlève de son chemin. Je me console. Pronominal qui vient bien sûr de l'Histoire et des histoires, du discours mettons. Mais dans cette distance à soi, dans cette saisie de soi comme distance des autres (on n'aura point à trop se forcer pour voir ici: Je sens le fond de miroir, les métaphores, dit sinon fait par le texte, tel fameux stade du bon docteur Jacques), dans ce clivage du sujet surgit le dit du désir: L'oreille collée sur le cadavre d'un voyeur, / Je sens le fond de miroir, les métaphores, / La poésie allumée, la poésie éteinte. / Je vois ma relation avec cette jeune femme comme une mission à l'étranger.

Dans le battement (la poésie allumée, la poésie éteinte) et l'intermittence qui, avec leur différance: le clivage, la séparation, forment le dit fondamental (et fondateur?) de ce poème, verra-t-on l'exil insurmontable d'Edipe ou l'un des célibataires (Célibataire afin de durer mon temps parmi les autres célibataires), pris dans le miroir, moulé mâlique, témoin oculiste, de l'éternelle mariće de Duchamp?

Si le texte de Des Roches emprunte ici parfois sa configuration à deux des signifiants majeurs de notre siècle, c'est que le simulacre biographique, ce semblant de vie qui nous fait écrire, est toujours par le fait même un intertexte, que Freud et Duchamp notamment disent peut-être, par leurs auvres, une métaphore de l'intertexte que déclenche l'Un du philosophe, et que toute intimité n'est jamais qu'allusive.

21 Je lui ai déclaré, avec le sourire,

Que la poésie est essentiellement pronominale. (Strophe 5, p. 12) 
Cette œuvre repose, et c'est sa modernité incontournable, la question, ancestrale et inouïe encore, des lieux du «Logos», de ses modes: philosophique, scientifique, religieux, littéraire... Sa sagesse est le fruit de sa science, de son écriture. Car, comme disait Heidegger: Le langage est beaucoup plus pensant et plus ouvrant que nous 22 .

En acceptant de s'ouvrir, d'ouvrir sa vie au langage qui le, la, nous clive, RDR, écrivant, a pensé loin.

Et si l'on voulait, pour mémoire et pour l'anthropométrie, redresser, positiviser, jovialement, l'énoncé ironique de Normand de Bellefeuille: Ceci n'est pas un texte important, mais roux 23 , il faudrait, il vaudrait mieux écrire: Ceci est un texte blond vénitien, mais important. fait.

Et finir, en pirouette, sur cette citation qui, bien sûr, n'en est pas une tout à

22 Martin Heidegger, Héraclite, Séminaire du semestre d'hiver 1966-1967 (en collaboration avec Eugen Fink), Paris, Gallimard, 1969, (coll. «Classiques de la philosophie»), p. 176.

23 Il s'agit du titre d'une des «parties» de Pourvu que ça ait mon nom (op. cit.), et plus précisément du texte qui s'inscrit justement en face de celui de Des Roches qui sert d'éponyme au recueil. L'allusion biographique est ici transparente, pour qui, il est vrai, connaît l'individu RDR, que l'on dit généralement «roux» avant qu'il ne vous reprenne: «blond vénitien». Mais précisément l'œuvre entière est aussi, pour des cercles restreints mais changeants, au point que nul n'a jamais la totalité de l'allusion, une manière de "private joke». 